\title{
Design and optimization of cost-effective coldproof portable enclosures for polar environment
}

\author{
Behzad Parsi, Lihong Zhang \\ Department of Electrical and Computer Engineering, Faculty of Engineering and Applied Science, \\ Memorial University of Newfoundland, Canada
}

\section{Article Info \\ Article history: \\ Received Jan 2, 2020 \\ Revised May 28, 2020 \\ Accepted Jun 6, 2020}

\section{Keywords:}

Cold environment enclosure Finite element method (FEM) 3D printing prototype Fuzzy logic control (FLC) Thermal resistor

\begin{abstract}
Based on the International Electrotechnical Commission standards, the electronic devices in the industrial class (e.g., integrated circuits or batteries) can only operate at the ambient temperature between $-40^{\circ} \mathrm{C}$ and $85^{\circ} \mathrm{C}$. For the human-involved regions in Alaska, Northern Canada, and Antarctica, extreme cold condition as low as $-55^{\circ} \mathrm{C}$ might affect sensing electronic devices utilized in the scientific or industrial applications. In this paper, we propose a design and optimization methodology for the selfheating portable enclosures, which can warm up the inner space from $-55^{\circ} \mathrm{C}$ for encasing the low-cost industrial-class electronic devices instead of expensive military-class ones to work reliably within their allowed operating temperature limit. Among the other options, ceramic thermal resistors are selected as the heating elements inside the enclosure. The placement of the thermal resistors is studied with the aid of thermal modelling for the single heating device by using the curve fitting technique to achieve uniform temperature distribution within the enclosure. To maintain the inner temperature above $-40^{\circ} \mathrm{C}$ but with the least power consumption from the thermal resistors, we have developed a control system based on the fuzzy logic controller. For validation, we have utilized COMSOL Multiphysics software and then one prototyped enclosure along with the fuzzy control system. Our experimental measurement exhibits its efficacy compared to the other design options.
\end{abstract}

Copyright (C) 2020 Institute of Advanced Engineering and Science. All rights reserved.

\section{Corresponding Author:}

Behzad Parsi,

Department of Electrical and Computer Engineering,

Faculty of Engineering and Applied Science,

Memorial University of Newfoundland,

St. John's, Canada NL A1B 3X5.

Email: bparsi@mun.ca

\section{INTRODUCTION}

An important factor for the normal operation of electronic devices is their ambient temperature. Low temperature may change the property of material and then vary the electrical resistivity. The resistivity of intrinsic semiconductor materials increases at a lower temperature, while extrinsic semiconductor materials have more complicated temperature profiles. As a result, any change in the ambient temperature can cause variations in threshold voltage, saturation velocity, and carrier mobility of a MOSFET [1,2].

Kumar and Kursun showed that the propagation delay of various logic gates increased up to $19.6 \%$ in $180 \mathrm{~nm}$ CMOS technology and $54.5 \%$ in $65 \mathrm{~nm}$ CMOS technology when the temperature rose from $25^{\circ} \mathrm{C}$ to $125^{\circ} \mathrm{C}$ [3]. To address the thermophysical effects of the state-of-the-art three-dimensional integrated circuits (ICs), comprehensive analysis and optimization of the critical structures including die, device layer, heat sink, and heat spreader were conducted in [4]. On the other hand, when the temperature is very low, semiconductors would turn to insulators, which do not feature conductance any longer. Therefore, ICs must 
be warmed up first to allow bandgap voltage references to boot. Keane et al. claimed that low temperature of $-50^{\circ} \mathrm{C}$ or below might terminate the normal operation of switches, relays, ICs, and other part of electrical equipment [5]. They suggested that electronic devices should work at the temperature of $-25^{\circ} \mathrm{C}$ or above if no heating unit is used.

Based on the International Electrotechnical Commission (IEC) standards, the electronic devices in the industrial/commercial class can only operate at the temperature between $-40^{\circ} \mathrm{C}$ and $85^{\circ} \mathrm{C}$. If any electronic device has to work below such an allowed temperature range, there may be the following two options. The first countermeasure is to use military-class electronic devices, which are normally highly expensive. As a second choice, a heating method has to be deployed to warm up the local surroundings of ICs or batteries to above $-40^{\circ} \mathrm{C}$ if the ambient temperature stays lower.

In the recent years, many new methods have been introduced for warming up batteries. Ji and Wang compared different schemes (such as internal heating, external heating, and convection heating) in terms of warm-up time, capacity loss, system durability, cost, and lifetime [6]. They showed that the heating time could be significantly shortened with those methods especially when reducing cell output voltage. They also claimed that the battery capacity loss was around 5\% if using the high-efficiency heating method. Later Wang et al., proposed a new lithium-ion battery structure for self-heating to address its power loss in cold climates [7]. This mechanism can internally warm up battery to work normally within 20 seconds at $-20^{\circ} \mathrm{C}$ and 30 seconds at $-30^{\circ} \mathrm{C}$. More recently, Guo et al., proposed an electro-thermal coupled model-based computational method for accurately estimating the battery thermal behavior [8]. It has the potential of balancing heat generation rate and battery lifetime degradation for reaching the optimal preheating performance. Nevertheless, to the best of our knowledge, thus far there is no reported work in the literature that can sustainably warm up any industrial/commercial-class electronic devices working in the extremely cold polar environment.

Different from classical or digital logic operating on discrete values (either 0 or 1 ), a fuzzy control system is based on fuzzy logic by analyzing continuous analog input values in terms of logical variables between 0 and 1 . By providing a means of converting linguistic control strategies, the fuzzy logic control method is especially suitable for the systems, which cannot be easily mathematically modeled [9]. The fuzzy logic controller (FLC) consists of four components: fuzzification interface, knowledge base, inference engine, and a defuzzification interface [10]. Each of these components has a different role in the control process and thus affects the performance of the whole system. In [11], Parsi et al., proposed a FLC to accurately control temperature in a cost-effective disposable off-chip micro-dispenser. A Peltier thermoelectric device supported by the FLC can warm up or cool down liquid for droplet transportation in the $\mu \mathrm{L}$ range, along with an electromagnetic actuator.

In this paper, we propose a design methodology of external portable structures (called enclosures hereafter) in the extremely cold environment (up to $-55^{\circ} \mathrm{C}$ ) to warm up any internal industrial-class low-power electronic systems, which may consist of ICs, sensors, and batteries normally operating at the temperature between $-40^{\circ} \mathrm{C}$ and $85^{\circ} \mathrm{C}$ [12-14]. For choosing the external heating sources, ceramic heating elements are studied both by using COMSOL Multiphysics software and experimentally [15, 16]. We have also developed a control system based on fuzzy logic to control inner temperature and reduce power consumption.

This paper is organized as follows. In section 2, the design constraints and optimization strategies for the type, number, location/style, and placement of heating elements will be studied. An analytic modeling method will be presented in section 3 to simplify the enclosure design procedure. Our proposed fuzzy logic controller will be discussed as well. In section 4 we will report our experimental results. Finally, the conclusion is drawn in section 5 .

\section{STUDY OF HEATING ELEMENTS}

In general, there are various electrical devices that can be used as electric heaters. Some of these heating devices in the industrial/commercial applications include incandescent light bulbs, thermal resistors, and thermoelectric devices. In this research, energy conversion efficiency, simplicity, and low cost are the major criteria that we have applied in the component selection.

An incandescent light bulb normally converts less than 5\% of the energy into visible light. A study has shown that this number for a standard light bulb is only about $2.2 \%$, while $97.8 \%$ of the energy is actually converted to heat [17]. That is to say, the incandescent light bulb may be used as a fair heat source. Another source of heat is the ceramic heating elements, e.g., ceramic thermal resistors that are both simple and inexpensive widely available in the typical electrical laboratories. Each ceramic thermal resistor is made of a small cylinder of graphite, surrounded by a protective ceramic layer. Short wires, or leads, are attached to both ends of the graphite cylinder and held in place by the coating material. A thermoelectric device, 
as the third type of electric heating elements, can actually offer more functions. In contrast to the incandescent light bulbs and ceramic heating elements, its main advantage is that the low-temperature surface would absorb heat and the high-temperature surface would release heat when electrical current passes through the device in the opposite directions. If the direction of the electrical current changes, the hot and cold surfaces would actually interchange [18]. In other words, the direction of heat dissipation is completely reversible so that a heating device can be changed to a cooling device if the polarity changes.

The main purpose of this study is to design cost-effective enclosures for polar environmental conditions. In this regard, an inexpensive electric heater has to be selected. In general, the cost of a thermoelectric device is 35 times more than that of a thermal resistor, while an incandescent light bulb is within the same price range as a thermal resistor. In a demonstrative experiment, we conducted finite element method (FEM) simulation to compare the efficiency of the ceramic thermal resistor unit and the incandescent light bulb unit under the same conditions (i.e., the same total power consumption for both heating units in addition to the same environmental condition such as the same initial temperature $-55^{\circ} \mathrm{C}$ and the same surroundings). According to our experiment, the ceramic thermal resistor unit could heat up the enclosure more quickly than the incandescent light bulb unit if energizing both units with the same amount of power. As a result, the ceramic thermal resistors, as a better option in terms of heating efficiency, simplicity, and cost, are selected as the heating elements in this work.

Besides the type of the heating elements, the number and placement of the thermal resistors have to be also studied. The placement study is to identify the best location and define the constraints for orientating the heating elements to warm up any enclosure with the best thermal effect. Below our study will be mainly focused on three aspects: the number of the heating elements, the location/style of the heating elements, and the placement constraints [19].

First of all, we conduct several FEM simulations to study whether one single or multiple heating elements (given the identical electrical power) would lead to better thermal effect. This case study is based on an enclosure with the dimension of $10 \mathrm{~cm} * 10 \mathrm{~cm} * 10 \mathrm{~cm}$ (for width, length, and height, respectively). In addition, an insulated boundary condition is added to the FEM model to decrease the heat flux leakage to the ambient, whose temperature is set as $-55^{\circ} \mathrm{C}$ constant. Moreover, constant heat sources are added to the model. The initial ambient and inside temperature of the enclosure is $-55^{\circ} \mathrm{C}$. Upon the initial conditions, the simulation is set to run 150 minutes.

By assuming constant total power consumption, a comparison between the heating efficiency and the number of the deployed thermal resistors is studied. The temperature average and standard deviation are listed in Table 1. As can be observed from the table, the enclosure inner space cannot be warmed up uniformly if using too fewer thermal resistors. But when five $1 \mathrm{~W}$ thermal resistors are used, the average inner space temperature reaches $-33.37^{\circ} \mathrm{C}$, which is less than $0.2 \%$ compared to that achieved by the four $1.25 \mathrm{~W}$ thermal resistors. Meanwhile adding one more thermal resistor would incur more cost in addition to more space. As a result, a placement of four thermal resistors along each side is the best arrangement for uniformly warming up the inner space of the enclosure in this case study.

Table 1. The average temperature and standard deviation for 18 sampling points

\begin{tabular}{cccccc}
\hline Number of Thermal Resistors & 1 & 2 & 3 & 4 & 5 \\
\hline Power of Each Thermal Resistor & $5 \mathrm{~W}$ & $2.5 \mathrm{~W}$ & $1.66 \mathrm{~W}$ & $1.25 \mathrm{~W}$ & $1 \mathrm{~W}$ \\
Average Temperature & $-36.43^{\circ} \mathrm{C}$ & $-35.46^{\circ} \mathrm{C}$ & $-34.75^{\circ} \mathrm{C}$ & $-33.42{ }^{\circ} \mathrm{C}$ & $-33.37^{\circ} \mathrm{C}$ \\
Temperature Standard Deviation. & $8.6749^{\circ} \mathrm{C}$ & $6.2694^{\circ} \mathrm{C}$ & $5.8429^{\circ} \mathrm{C}$ & $2.9726^{\circ} \mathrm{C}$ & $2.5982^{\circ} \mathrm{C}$ \\
\hline
\end{tabular}

Another important issue for the heating element placement is to understand the constraints with reference to the electronic devices accommodated in the $3 \mathrm{D}$ enclosure. A $2 \mathrm{D}$ analysis shows that the best location of the heating elements is along the sides, while in the 3D model the heating elements may be placed on the top, bottom, or in the middle of the enclosure. Therefore, another group of simulation is executed to find out the effective distance from a thermal resistor. The effective distance stands for the distance from a unit power thermal resistor that can warm up the surrounding operational elements (e.g., printed circuit boards or batteries) without causing damage. By using curve fitting technique [20, 21], the best approximation of a linear model based on a series of sampled data points can be obtained. As can be seen in Figure 1(a), the radial effective distance $d_{r}$ (i.e., the vertical one in the 2D view) and axial effective distance $d_{a}$ (i.e., the horizontal one in the 2D view) for heating up the surrounding objects are between zone 2 and zone 5 (i.e., $l / 9<d_{r}<l / 2.3$ and $w / 6.4<d_{a}<w / 1.6$ where $l$ and $w$ are the thermal resistor device length and width respectively on the facing side). The root mean square (RMS) error is also listed in Figure 1(a). As a result, the best place of locating a thermal resistor unit is in the middle since it can distribute the heat in both directions. 
Another point that can be observed from Figure 1(a) is that the operational elements must not be placed in zone 1 (i.e., very close to or overlapping with the heating elements), which has the highest temperature compared to the other zones. Instead they should be located within the effective distance with reference to the thermal resistor that is located in the middle of Figure 1(a). The enclosure inner space should be warmed up uniformly. Otherwise, uneven warmup may cause serious damage due to the thermal stress that would be applied to the internal printed circuit boards or batteries accommodated inside the enclosure. Certain cracks might be propagated along the electronic devices to eventually cause open circuit problems [22].

The heating conduction along the radius of thermal resistors' carbon cylinder, which eventually leads to the radial effective distance as shown in Figure 1(a), is much more than the axial one because of larger surface contact with the coating layer. As a result, the area determined by the effective distance in Figure 1(a) has an approximately rectangle shape instead of a square one. Figure 1(b) shows the average temperature of 18 sampling points inside zones 1-6 in the 3D model of the enclosure as a function of time. The points, which are selected at $l / 9<d_{r}<1 / 2.3$ and $w / 6.4<d_{a}<w / 1.6$ (i.e., within zones 2-5), have an average temperature above $-40^{\circ} \mathrm{C}$ at the steady state (i.e., after passing the first 150 minutes), whereas the points located elsewhere are either too cold or unnecessarily too warm. In addition, we conduct another group of FEM simulation to study whether the size of the enclosure may change the effective distance of one-unit thermal resistor. We deploy two-unit thermal resistors in two different size enclosures with the same environmental condition, each of which consumes the same electrical power. Our study shows that the effective distances of two thermal resistors bear no change from each other due to the enclosure size change.

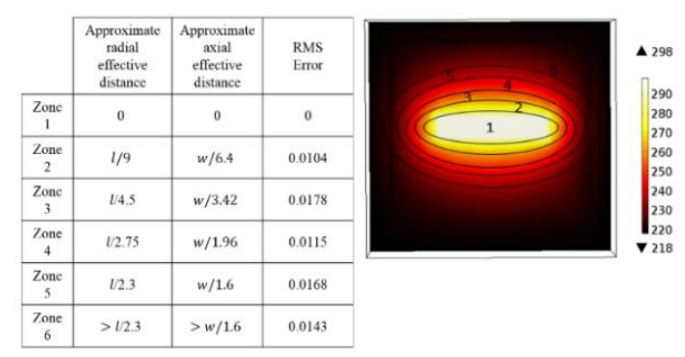

(a)

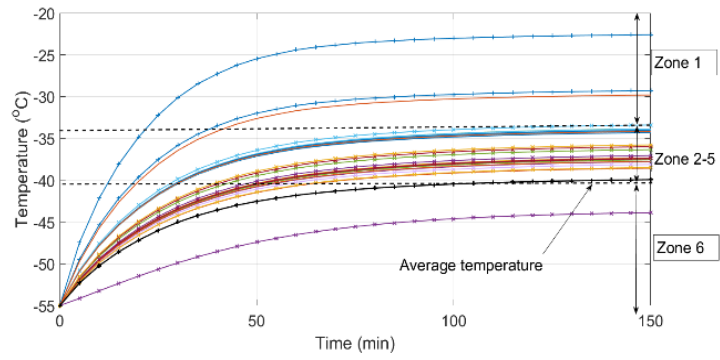

(b)

Figure 1. (a) The effective distance of a unit power thermal resistor ( $l$ and $w$ are the length and width of the thermal resistor) located in the middle, (b) The temperature plot as a function of time for 18 sampling points inside zones 1-6

\section{ANALYTIC MODELING AND FUZZY LOGIC CONTROLLER}

The case-study tactics and design knowledge accumulated from the previous section are highly valuable in the practical design process so that the designers can work out a specific enclosure design without any further expensive FEM simulations once the thermal modeling of the unit thermal resistor is completed. Therefore, here we aim to optimize the number of thermal units to reduce power consumption and hardware cost, in addition to increasing the available space for holding more electronic components inside the enclosure. To reach this objective, we are motivated to study how to model the relationship between the required power and the number of unit thermal resistors in the optimization process with a constraint of maintaining the average inner temperature of the enclosure above $-40^{\circ} \mathrm{C}$ in the polar environment. Note that such a threshold temperature of $-40^{\circ} \mathrm{C}$ can be changed to another user-defined number as long as the thermal modeling of the unit thermal resistor is conducted in advance accordingly. Based on the concept of effective distance supported by the curve fitting technique detailed in Section 2, we propose a general analytic design methodology as follows, with which the designers can quickly estimate the required power and thermal resistor number for heating up the enclosure to above $-40^{\circ} \mathrm{C}$ without any expensive FEM simulations. Figure 2(a) shows the schematic view of the enclosure with unit thermal resistors in the row-based configuration, which is aimed for the enclosures with relatively larger geometry size (with reference to the size of the unit thermal resistor). In contrast, Figure 2(b) shows the schematic view in the side-based configuration, which is dedicated to the enclosures with smaller geometry size. Figure 2(c) depicts the effective distance of each unit thermal resistor.

To establish the relationship between the number of unit thermal resistors and enclosure size, we should formulate a set of analytic functions [23]. By considering the size limitation of the unit thermal 
resistor in addition to the maximum effective distance of each thermal resistor, we have set up two different models to identify the best location for orientating the heating elements to warm up the enclosure with the best thermal effect. We have utilized a user-defined threshold value, which is equal to $2 L_{r}$ by default, to determine which model should be selected. In other words, if each dimension of the enclosure is less than $2 L_{r}$, the side-based configuration will be utilized so that each thermal resistor will be placed near each side for warming up the inner space of the enclosure uniformly. Otherwise, the following functions will be utilized:

$$
\begin{aligned}
& A_{1}=\left(W_{c}-2 t\right)\left(L_{c}-2 t\right), \quad A_{2}=\left(L_{r}+1.256 W_{r}\right)\left(W_{r}+0.869 L_{r}\right), \quad A_{1}=m A_{2}, \\
& m=\frac{\left(W_{c}-2 t\right)\left(L_{c}-2 t\right)}{\left(L_{r}+1.256 W_{r}\right)\left(W_{r}+0.869 L_{r}\right)} \text { if }\left(L_{c}-2 t\right)>2 \mathrm{~L}_{\mathrm{r}} \text { and }\left(W_{c}-2 t\right)>2 \mathrm{~W}_{\mathrm{r}},
\end{aligned}
$$

where $W_{c}$ and $L_{c}$ are the width and length of the enclosure, $t$ is the thickness of the insulation layer, $W_{r}$ and $L_{r}$ are the width and length of each unit thermal resistor respectively, and $m$ is the number of the unit thermal resistors in the $2 \mathrm{D}$ view of the enclosure.

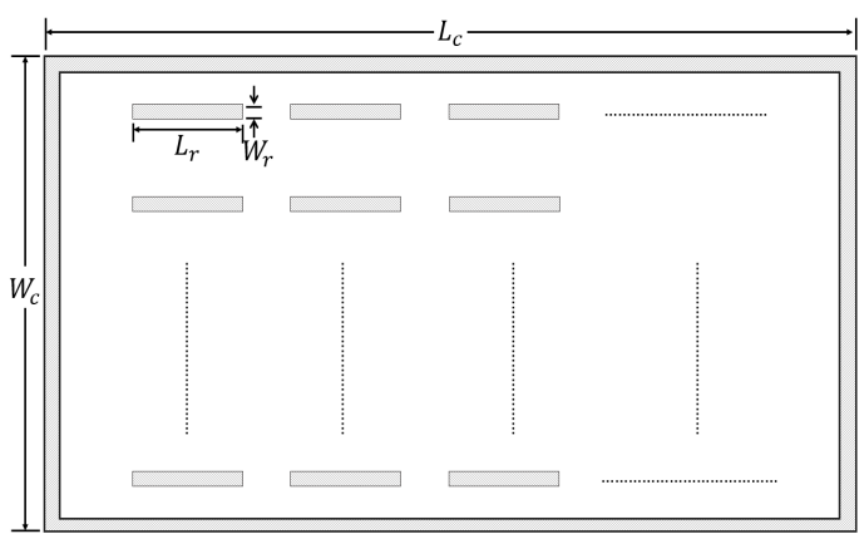

(a)

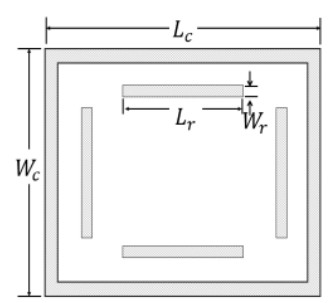

(b)

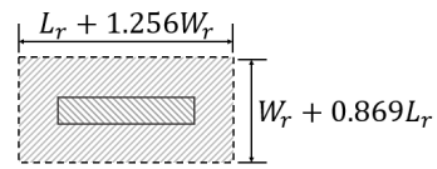

(c)

Figure 2. The schematic view of the enclosure with unit thermal resistors (a) in the row-based configuration and (b) in the side-based configuration, with the effective distance (c) around a unit thermal resistor

With the same tactic, for the 3D modeling, the number of the levels in $\mathrm{Z}$ direction can be found as:

$$
k=\frac{H_{C}-2 t}{0.869 L_{r}+W_{r}},
$$

where $H_{c}$ is the height of the enclosure. In all, the total required integer number of the unit thermal resistors is:

$$
N=\lceil m * k\rceil,
$$

where a ceiling function $(\lceil\rceil)$ is applied. Accordingly, the total required power, which ensures the average inner temperature of the enclosure is always above $-40^{\circ} \mathrm{C}$, can be expressed as:

$$
P=N * P_{\text {unit }},
$$

where $P_{\text {unit }}$ is the power of the unit thermal resistor. The estimation above would help a designer to evaluate the number of the required thermal resistors and the corresponding required power without invoking any expensive FEM simulations [24].

Autonomously controlling inner temperature of the enclosure is of importance in this research since in the remote area the enclosures have to detect the ambient temperature and automatically tune their thermal units. In this study, we have utilized the fuzzy logic control method for the temperature control of the enclosures. In this regard, the required power that the thermal resistor units need to heat up the enclosure is the input of our target system to be controlled, while the temperature that is read out by the temperature 
sensors is the output of our target control system. Table 2 lists the rule base of our developed fuzzy logic controller. The linguistic input terms include very negative, negative, zero, positive, very positive, while the linguistic output variables include zero, very low, low, high, very high.

Table 2. The rule base of the developed fuzzy logic controller

Controller Rule 1: If the temperature difference is very negative, then the output power must be zero
Controller Rule 2: If the temperature difference is negative, then the output power must be very low
Controller Rule 3: If the temperature difference is zero, then the output power must be low
Controller Rule 4: If the temperature difference is positive, then the output power must be high
Controller Rule 5: If the temperature difference is very positive, then the output power must be very high

Figure 3(a) shows the input of the fuzzy sets, which is defined as the temperature difference between the reference temperature (i.e., $-40^{\circ} \mathrm{C}$ ) and the actual sampled temperature. Based on the input fuzzy terms, our developed fuzzy control rule base can derive an appropriate output fuzzy set. Then a corresponding power value can be generated as shown in Figure 3(b).

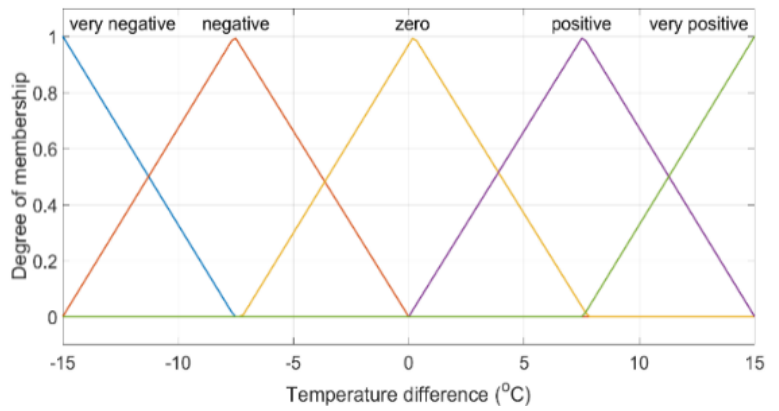

(a)

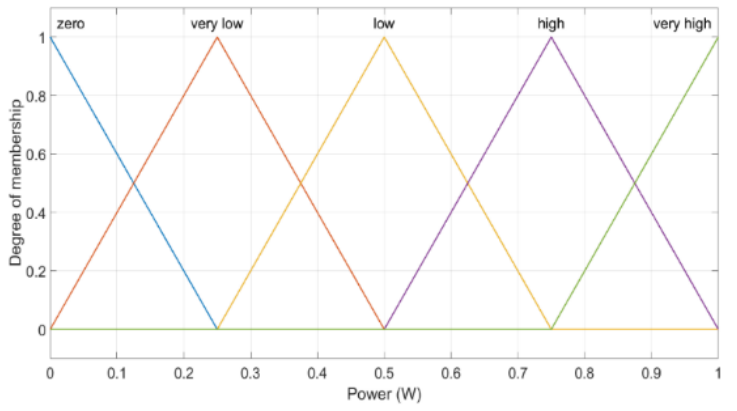

(b)

Figure 3. (a) Correspondence between linguistic terms and fuzzy input values,

(b) Correspondence between membership functions and fuzzy output sets

\section{EXPERIMENTS AND DISCUSSION}

By following the case study discussed in Section 2, we prototyped the enclosure with a 3D printer (Formlabs Form 2SLA). Figure 4 shows this prototype, which is made of polycarbonate. To make sure that our enclosure has the minimum heat flux leakage, we need to form proper insulation around the enclosure. Among the different options, foam insulation has become the most widely used insulation method. In this work, we select polyurethane (PU) foam as an insulation layer thanks to its low thermal conductivity and low cost in addition to ease of processing. In this experiment, $W_{c}, H_{c}$, and $L_{c}$ are all equal to $10 \mathrm{~cm}$. In addition, $W_{r}$ and $H_{r}$ are equal to $1 \mathrm{~cm}$, while $L_{r}$ is equal to $4.5 \mathrm{~cm}$. As a result, $\left(L_{c}-2 t\right)$ is equal to $8 \mathrm{~cm}$, which is less than the threshold value of $2 L_{r}=9 \mathrm{~cm}$. Thus, the side-based configuration was utilized. It means that each thermal resistor is placed along each side of the enclosure.
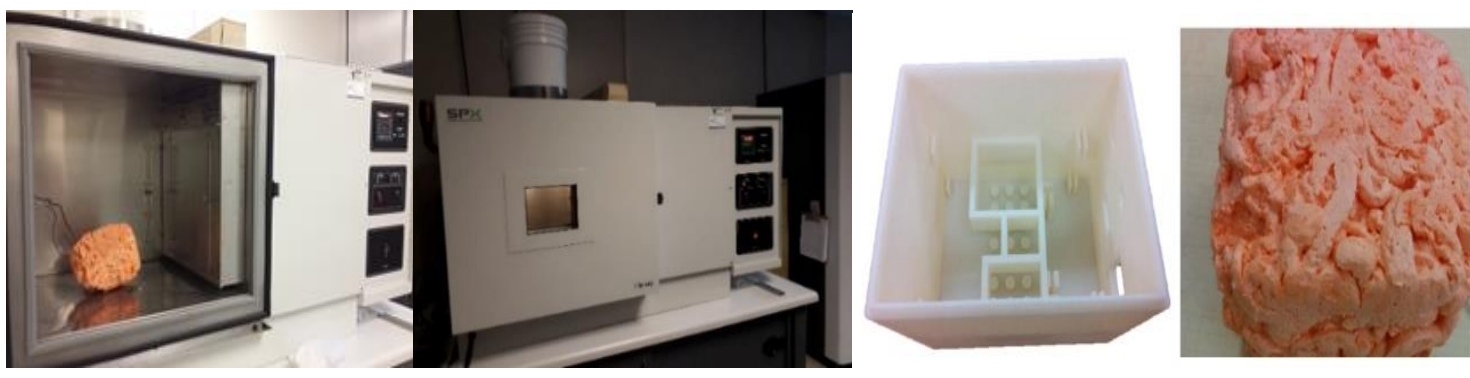

Figure 4. Cold chamber setup and the enclosure prototype with an insulation layer 
The enclosure was put into a cold chamber to have its ambient temperature of $-55^{\circ} \mathrm{C}$ as shown in Figure 4. Then the enclosure started to warm up and the inner temperature was recorded. To measure the inner temperature of the enclosure, four temperature sensor DS18B20 (with a measurement range of $-55^{\circ} \mathrm{C} \sim+125^{\circ} \mathrm{C}$ featuring strong disturbance resistance and high precision) were utilized to measure the average of the inner temperature inside the enclosure.

In order to maintain the average temperature of the enclosure at $-40^{\circ} \mathrm{C}$ or above, our proposed fuzzy control logic, which is detailed in Section 3, was utilized in our experiments. Figure 5(a) illustrates the schematic of the close-loop testbench with our proposed fuzzy logic controller and enclosure prototype. The reference temperature of the fuzzy logic controller was set as $-40^{\circ} \mathrm{C}$. Figure $5(\mathrm{~b})$ shows the average temperature of the enclosure prototype when the fuzzy logic controller with a digital filter was utilized. As can be seen from this figure, the settling time slightly decreased and the actual average temperature was securely bound to the reference temperature compared to the open-loop system as shown in Figure 1(b). This shows the high performance of our proposed enclosure heating and control system, which can reach the target temperature faster and then stays there with a smoother curve for saving energy.

To compare the efficiency of our proposed fuzzy logic controller with the other alternatives, we also included state machine controller and PID controller in our experiments by using the same testbench shown in Figure 5(a). Similar to the fuzzy logic controller, these two general controllers are widely utilized for the systems, which cannot be readily mathematically expressed [25]. The state machine controller constructs a reactive system, which consists of a finite number of states and changes states as per the given conditions. We implemented it by defining two states and switching them upon a comparison between the sensed temperature from the DS18B20 temperature sensor and the reference temperature $\left(-40^{\circ} \mathrm{C}\right)$. As for the PID controller widely used in industrial control systems, a control loop mechanism is built up to employ feedback for continuously modulated control. Normally the PID controller continuously calculates an error value as the difference between a desired reference point and a measured process variable. It applies a correction based on proportional, integral, and derivative terms (denoted by $P, I$, and $D$ respectively just as the acronym of the controller). In our implementation, three operators (i.e., proportional, integral, and derivative) are defined to represent the modulated error between the sampled temperature from the DS18B20 temperature sensor and the reference temperature.

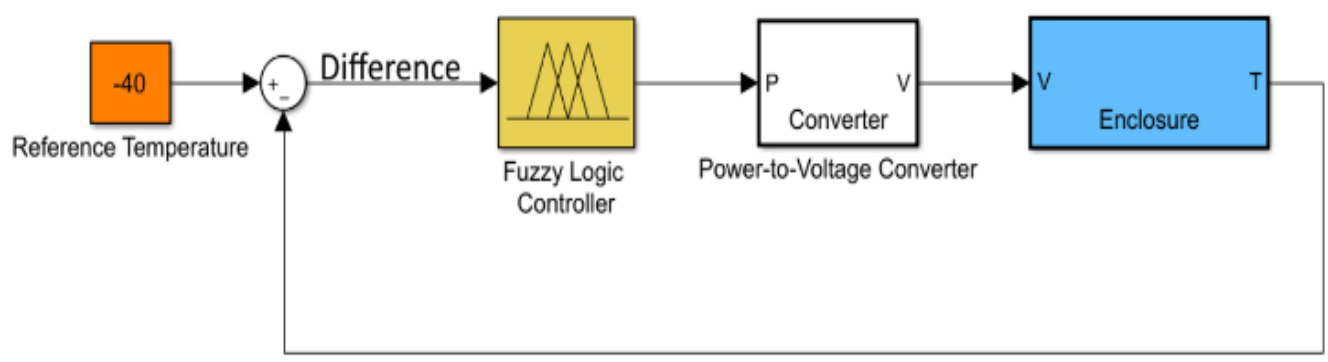

(a)

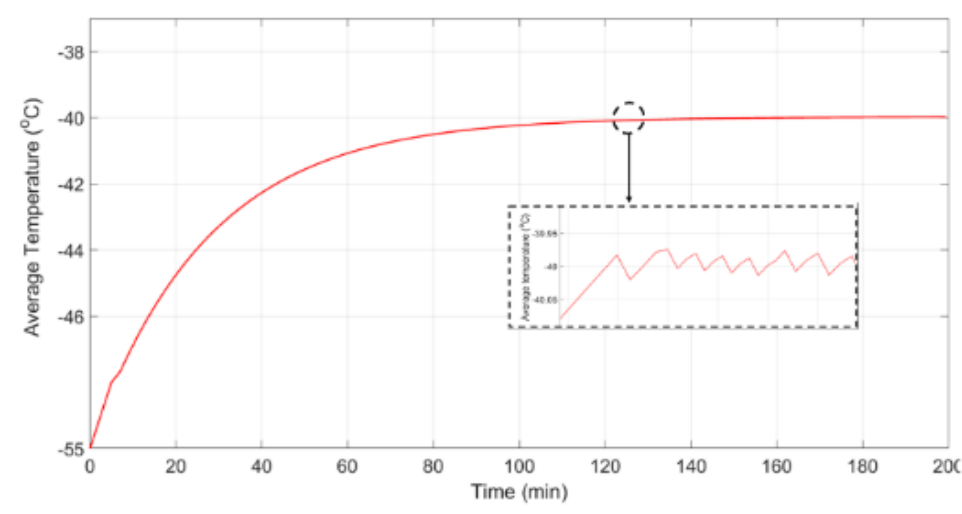

(b)

Figure 5. Fuzzy logic controller (FLC), (a) The schematic of the close-loop system with the proposed FLC, (b) The average temperature of the enclosure prototype (driven by the proposed FLC) versus time 
Figure 6(a) shows the comparison among the state machine, PID, and our proposed fuzzy logic controllers. As can be seen from this figure, the output from the fuzzy logic controller has the least oscillation around the reference temperature since the output power can continuously change between 0 and 1 . In contrast, the state machine controller suffers large fluctuation $\left( \pm 0.26^{\circ} \mathrm{C}\right)$ around the reference temperature of $-40^{\circ} \mathrm{C}$. This is mainly due to the simple control logic used in this controller. Although the PID controller could work better than the state machine controller in terms of temperature smoothness segmentally, the magnitude of the temperature rippling $\left( \pm 0.31^{\circ} \mathrm{C}\right)$ along the reference temperature was actually even larger. As for our proposed fuzzy logic controller, the average oscillation around the reference temperature $\left(-40^{\circ} \mathrm{C}\right)$ was only $\pm 0.02^{\circ} \mathrm{C}$.

To compare the power consumption, the outputs of the system for the state machine, PID, and fuzzy logic controllers are plotted in Figure 6(b). To calculate the total power consumption within the unit time (e.g., one hour), (6) is utilized and the results are summarized in Table 3. One can observe that, in the steadystate period, the total power consumption of our proposed fuzzy logic controller system is $17.6 \%$ less than the state machine controller and $38.04 \%$ less than the PID controller.

$$
P_{t}=\int_{t=0}^{n} P d t
$$

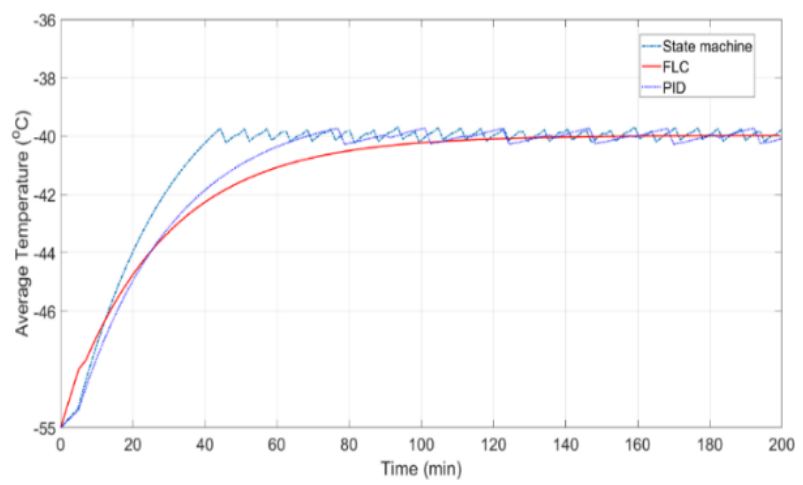

(a)

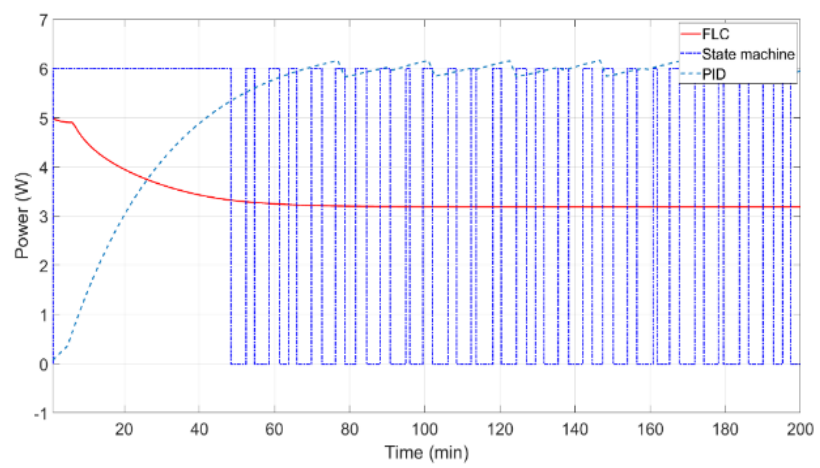

(b)

Figure 6. Comparison of (a) the average temperature versus time and

(b) the power outputs among the state machine controller, PID, and fuzzy logic controller (FLC).

Table 3. The total power consumption (WH)

\begin{tabular}{ccccc}
\hline Time $(\mathrm{min})$ & 60 & 120 (steady-state) & 180 (steady-state) & 240 (steady-state) \\
\hline State machine & 5.6173 & 9.4640 & 13.3277 & 17.1672 \\
PID & 3.5567 & 9.9767 & 16.3967 & 22.8267 \\
Fuzzy logic controller (FLC) & 4.8121 & 7.9197 & 11.0997 & 14.1495 \\
\hline
\end{tabular}

\section{CONCLUSION}

In this paper we presented a complete design methodology for cost-effective portable enclosures, which can accommodate industrial-class electronic devices operating at the ambient temperature as low as $-55^{\circ} \mathrm{C}$. Among the alternatives, we selected ceramic thermal resistors as the heating elements inside 
the enclosure. Moreover, the optimal number, location/style, and the placement constraints of the heating elements were studied. Based on the thermal modeling of the unit heating elements by using curve fitting technique, we proposed an analytic model, which can be used to estimate the number of the required thermal resistors or the total required power in the practical enclosure design. In addition, we developed a fuzzy logic controller to manipulate the inner temperature of the enclosure. Our experimental results demonstrated that the prototype enclosure with the proposed fuzzy logic controller can achieve excellent performance compared to the other alternatives.

\section{REFERENCES}

[1] X. Dong and L. Zhang, "EA-based LDE-aware fast analog layout retargeting with device abstraction," IEEE Transactions on Very Large Scale Integration Systems, vol. 27, no. 4, pp. 854 - 863, 2019.

[2] L. Zhang, "VLSI circuit layout," Wiley Encyclopedia of Computer Science and Engineering, vol. 5, pp. 3034-3044, 2009.

[3] R. Kumar and V. Kursun, "Impact of temperature fluctuations on circuit characteristics in $180 \mathrm{~nm}$ and $65 \mathrm{~nm}$ CMOS technologies," in IEEE Int. Symp. on Circuits and Syst., pp. 3858-3861, May 2006.

[4] F. Tavakkoli, S. Ebrahimi, S. Wang, K. Vafai, "Thermophysical and geometrical effects on the thermal performance and optimization of a three-dimensional integrated circuit," ASME Journal of Heat Transfer, vol. 138, no. 8, pp. 082101:1-7, 2016.

[5] B. Keane, G. Schwarz, and P. Thernherr, "Electrical equipment in cold weather applications," in Industry Applications Society 60th Annual Petroleum and Chemical Industry Conference, pp. 1-7, 2013.

[6] Y. Ji and C.-Y. Wang, "Heating strategies for Li-ion batteries operated from subzero temperatures," Electrochimica Acta, vol. 107, pp. 664-674, 2013.

[7] C.-Y. Wang, G. Zhang, S. Ge, T. Xu, Y. Ji, X.-G. Yang, and Y. Leng, "Lithium-ion battery structure that self-heats at low temperatures," Nature, vol. 529, no. 7587, pp. 515-518, 2016.

[8] S. Guo, R. Xiong, K. Wang, and F. Sun, "A novel echelon internal heating strategy of cold batteries for all-climate electric vehicles application,” Applied Energy, vol. 219, pp. 256-263, 2018.

[9] W. B. Xie, Y. L. Wang, J. Zhang, and L. Huang, "Membership function dependent observer-based controller design for a t-s fuzzy system," Asian Journal of Control, vol. 19, no. 4, pp. 1496-1507, Jun. 2017.

[10] L. Zadeh and R. Aliev, "Fuzzy Logic Theory and Applications," World Scientific Publishing, 2018.

[11] B. Parsi, L. Zhang, and V. Masek, "Disposable off-chip micro-dispenser for accurate droplet transportation," IEEE Sensors Journal, vol. 19, no. 2, pp. 575-586, 2019.

[12] L. Zhang, V. Masek, and N. Sanatdoost, "Structural optimization of Z-axis tuning-fork MEMS gyroscopes for enhancing reliability and resolution," Microsystem Technologies, vol. 21, no. 6, pp. 1187-1201, 2015.

[13] S. Nabavi and L. Zhang, "Frequency tuning and efficiency improvement of piezoelectric MEMS vibration energy harvesters," IEEE Journal of Microelectromechanical Systems, vol. 28, no. 1, pp. 77 - 87, 2019.

[14] S. Nabavi and L. Zhang, "Design and optimization of MEMS piezoelectric energy harvesters for improved efficiency," in Proc. IEEE Sensors Conference, pp. 1-3, 2017.

[15] S. Nabavi and L. Zhang, "Design and optimization of a low-resonant-frequency piezoelectric MEMS energy harvester based on artificial intelligence," in Multidisciplinary Digit. Publishing Inst. Proc. (32nd Eurosensors Conf.), vol. 2, no. 13, pp. 1-5, 2018.

[16] B. Parsi, L. Zhang, and V. Masek, "Vibration analysis of a double circular PZT actuator for a valveless micropump," in CSME International Congress, May 27-30, pp. 17-24, 2018.

[17] N. Armaroli and V. Balzani, "Towards an electricity-powered world," Energy \& Environmental Science, vol. 4, no. 9, p. 3193, 2011.

[18] Y. Sheikhnejad, Z. Vujicic, Á. Almeida, R. Bastos, A. Shahparia, and A. Teixeira, "Analytical transient analysis of peltier device for laser thermal tuning," in 3rd Int. Conf. on Applications of Optics and Photonics, pp. 741-749, 2017.

[19] L. Zhang and U. Kleine, "A novel analog layout synthesis tool," in Proc. IEEE International Symposium on Circuits and Systems, pp. V101-V104, May 2004.

[20] T. Liao and L. Zhang, "Parasitic-aware gm/Id-based many-objective analog/RF circuit sizing," in Proc. IEEE International Symposium on Quality Electronic Design, pp. 100-105, 2018.

[21] Z. Zhao, T. Liao, and L. Zhang, "Fast performance evaluation for analog circuit synthesis frameworks," in Proc. IEEE International Symposium on Circuits and Systems, pp. 1-5, 2018.

[22] C. A. Boano, N. Tsiftes, T. Voigt, J. Brown, and U. Roedig, "The impact of temperature on outdoor industrial sensornet applications," IEEE Transactions on Industrial Informatics, vol. 6, no. 3, pp. 451-459, 2010.

[23] S. Nabavi and L. Zhang, "T-shaped piezoelectric vibratory MEMS harvester with integration of highly efficient power management system," in Journal of Physics: Conference Series (Power MEMS), vol. 1052, no. 1, pp. 1-4, 2018.

[24] S. Kaviani, M. Bahrami, A. Esfahani, and B. Parsi, "A modeling and vibration analysis of a piezoelectric micropump diaphragm," Comptes Rendus Mécanique, vol. 342, no. 12, pp. 692-699, 2014.

[25] T. Liao and L. Zhang, "Efficient parasitic-aware hybrid sizing methodology for analog and RF integrated circuits," Integration, the VLSI Journal, vol. 62, pp. 301-313, 2018. 